\title{
Identification of Ketene-Reactive Intermediate of Erlotinib Possibly Responsible for Inactivation of P450 Enzymes ${ }^{\mathbb{S}}$
}

\author{
Huimin Zhao, Siyuan Li, Zixin Yang, Ying Peng, Xiaohui Chen, and Jiang Zheng
}

School of Pharmacy (H.Z., X.C.) and Wuya College of Innovation (S.L., Z.Y., Y.P., J.Z.), Shenyang Pharmaceutical University, Shenyang, Liaoning, People's Republic of China; and State Key Laboratory of Functions and Applications of Medicinal Plants, Key Laboratory of Pharmaceutics of Guizhou Province, Guizhou Medical University, Guiyang, Guizhou, People's Republic of China (J.Z.)

Received November 3, 2017; accepted January 17, 2018

\begin{abstract}
Erlotinib (ELT), a tyrosine kinase inhibitor, is widely used for the treatment of nonsmall cell lung cancer in clinic. Unfortunately, severe drug-induced liver injury and other adverse effects occurred during the treatment. Meanwhile, ELT has been reported to be a mechanism-based inactivator of cytochrome P450(CYPs) 3A4 and 3A5. The objectives of this study were to identify ketene intermediate of ELT and investigate the association of the acetylenic bioactivation with the enzyme inactivation caused by ELT. A ketene intermediate was detected in human microsomal incubations of ELT, using
\end{abstract}

4-bromobenzylamine as a trapping agent. CYPs 3A4 and 3A5 mainly contributed to the bioactivation of ELT. Microsomal incubation study showed that the ketene intermediate covalently modified the enzyme protein at lysine residues and destroyed the structure of heme. The vinyl and ethyl analogs of ELT showed minor enzyme inhibitory effect (less than $\mathbf{2 0} \%$ ), whereas ELT inactivated more than $60 \%$ of the enzyme. The present study provided a novel bioactivation pathway of ELT and facilitated the understanding of the mechanisms of ELT-induced mechanism-based enzyme inactivation and liver injury.

\section{Introduction}

Erlotinib (ELT, 1; Scheme 1) is a highly potent reversible human epidermal growth factor receptor (EGFR) tyrosine kinase inhibitor (Dai et al., 2005). The proliferation and apoptosis of tumor cells are mediated by the EGFR signaling pathway (Veale et al., 1993; Scambia et al., 1995). Studies have shown that nonsmall cell lung cancer (NSCLC) was caused by overexpression of EGFR (Massarelli et al., 2013; Jotte and Spigel, 2015). ELT (Tarceva) was first approved by the Food and Drug Administration in 2004 for the treatment of metastatic NSCLC and advanced pancreatic cancer. Despite the beneficial effects of ELT, this antineoplastic drug has been linked to interstitial lung disease and hepatorenal syndrome (Tammaro et al., 2005; Makris et al., 2007). In addition, ELT has been associated with severe hepatic injury. The patients with normal liver function may suffer from hepatic impairment and even hepatic failure when treated with ELT (Liu et al., 2007; Saif, 2008; Huang et al., 2009). These rare but severe cases are often described as idiosyncratic toxicity. Although the mechanisms of ELTinduced hepatic injury remain unknown, it has been proposed that covalent modifications of cellular macromolecules by reactive metabolites might be responsible for the idiosyncratic adverse reactions (Stepan et al., 2011).

In humans, cytochrome P450 (CYPs) 3A4 and 3A5 are the critical enzymes in the liver metabolism of ELT, whereas CYPs 1A2 and 2C8 make a minor contribution (Ling et al., 2006). In addition, ELT has been proven to be a mechanism-based inactivator of CYPs 3A4 and 3A5 (Li et al., 2010). Mechanism-based inactivation (MBI) is oftentimes referred to as quasi-

This work was supported in part by the National Natural Science Foundation of China [Grants 81373471, 81430086, and 81773813].

https://doi.org/10.1124/dmd.117.079327.

S This article has supplemental material available at dmd.aspetjournals.org. irreversibly or irreversibly time-dependent inhibition, and/or suicide inactivation. Drug-induced MBI may cause more serious injuries to patients compared with reversible enzyme inhibition. The reason is that the MBI can destroy the structure of enzymes that would have to be regenerated, and loss of enzyme function may raise the possibility of unwanted drug-drug interactions. In theory, MBI arises when drugs are bioactivated by CYPs to generate reactive intermediates that cause irreversible loss of enzyme activity through covalent bonding to apoprotein and/or destroying structure of the heme (Kalgutkar et al., 2007; Orr et al., 2012).

As shown in Scheme 1, ELT (1) contains a phenyl-acetylene moiety. Metabolism of ELT is mediated by three primary routes in humans, as follows: 1) $O$-demethylation of the methoxy group of ELT; 2) 4-hydroxylation of the aromatic ring of aminophenylacetylene group to p-aminophenol; and 3) hydrolysis to phenyl acetic acid after oxidation of terminal acetylene (Ling et al., 2006). 4-Hydroxylation of the phenyl ring of ELT results in forming para-hydroxyaniline metabolite, which further undergoes oxidation to form a quinoneimine intermediate through loss of two electrons by CYPs. This quinoneimine intermediate has been detected as glutathione (GSH) conjugate by liquid chromatography/tandem mass spectrometry (Li et al., 2010).

Although the bioactivation of ELT by CYPs to a reactive quinoneimine metabolite has been well-defined, the possible bioactivation of this drug containing chemical scaffold terminal alkyne to a ketene intermediate is much less characterized. To our knowledge, the reactive oxirene and ketene intermediates can occur in the oxidation of the acetylene bond by CYPs and are thought to play a part in CYP inactivation (Blobaum et al., 2002; von Weymarn et al., 2004; Lin and Hollenberg, 2007). In this study, a novel bioactivation pathway of ELT in the form of an amino-group-based 4-bromobenzylamine (BBA)

ABBREVIATIONS: BBA, 4-bromobenzylamine; CYP, cytochrome P450; EGFR, epidermal growth factor receptor; ELT, erlotinib; GSH, glutathione; HLM, human liver microsome; HPLC, high performance liquid chromatography; LC-MS/MS, liquid chromatography-tandem mass spectrometry; $\mathrm{MBI}$, mechanism-based inactivation; $m$-CPBA, $m$-chloroperbenzoic acid; MRM, multiple reaction monitoring; MS/MS, tandem mass spectrometry; NSCLC, nonsmall cell lung cancer; PBS, potassium phosphate buffer. 


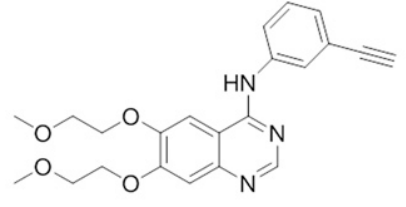

1

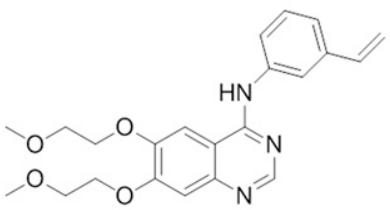

3

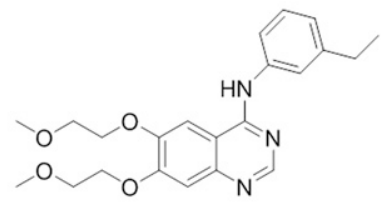

2

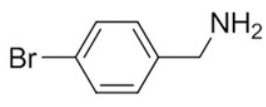

4
Scheme 1. Structures of ELT (1), ethyl analog (2), vinyl analog (3), and BBA (4).

adduct in NADPH-dependent human liver microsomes (HLMs) incubated with ELT was reported. Moreover, the correlation of ketene intermediate formation and enzyme inactivation was investigated.

\section{Materials and Methods}

Reagents. ELT ( $\geq 99 \%$ ) and chloroprotoferrihem were purchased from Dalian Meilun Biotech (Dalian, China). BBA was purchased from Shanghai Darui Chemical (Shanghai, China). $m$-Chloroperbenzoic acid ( $m$-CPBA), $10 \%$ palladium on activated charcoal, $10 \%$ palladium on barium sulfate, and quinoline were purchased from Aladdin Reagent (Shanghai, China). Pronase E, chymotrypsin, L-lysine, testosterone, $6 \beta$-hydroxytestosterone, DL-dithiothreitol, propranolol, and NADPH were purchased from Sigma-Aldrich (St. Louis, MO). Recombinant human P450 enzymes and HLMs were purchased from BD Gentest (Woburn, MA). Formic acid and trifluoroacetic acid were purchased from Fisher Scientific (Springfield, NJ). All organic solvents were obtained from Fisher Scientific. All chemicals were of analytical grade, and all solvents were of high performance liquid chromatography (HPLC) grade.

Chemical Synthesis of ELT Analogs. The ethyl and vinyl analogs (Scheme 1,2 and $\mathbf{3}$ ) were synthesized by catalytic hydrogenation of ELT, according to the published literature with modification (Ban et al., 2008). To synthesize the ethyl analog (2), ELT (100 $\mathrm{mg}, 0.26 \mathrm{mmol}$ ) was dissolved in $10 \mathrm{ml}$ methanol, and palladium on activated charcoal $(20 \mathrm{mg})$ was then added as a catalyst. The mixture was stirred in a sealed and hydrogen-purged reactor at room temperature and with pressure of $70 \mathrm{MPa}$ for 4 hours. The resulting reaction mixture was condensed under vacuum after filtration. Silica gel column chromatography with eluate of dichloromethane and methanol (20:1) was used to purify the product. The resulting product was characterized by HPLC-UV, tandem mass spectrometry (MS/MS), and ${ }^{1} \mathrm{H}$ NMR. Data and spectrums are shown in supporting information (Supplemental Figs. 1 and 2).

To synthesize the vinyl analog (3), ELT (100 $\mathrm{mg}, 0.26 \mathrm{mmol})$ was dissolved in $10 \mathrm{ml}$ methanol, and palladium on barium sulfate $(20 \mathrm{mg})$ was then added as a catalyst. Aliquots $(0.5 \mathrm{ml})$ of quinoline were added to quench the activity of the catalyst. The mixture was stirred in a sealed and hydrogen-purged reactor at room temperature and with pressure of $70 \mathrm{MPa}$ for 2 hours. After reaction, the mixture was subjected to a silica gel chromatography to remove quinolone, and the fraction was purified by a semipreparative HPLC system. The resulting product was characterized by HPLCUV, MS/MS, and ${ }^{1} \mathrm{H}$ NMR. Data and spectrums are shown in supporting information (Supplemental Figs. 3 and 4).

Microsomal Incubations. ELT (100 $\mu \mathrm{M}$ from a dimethylsulfoxide stock) and HLMs (1.0 mg protein/ml) were mixed in potassium phosphate buffer (PBS; $\mathrm{pH} 7.4$ ). BBA $(5 \mathrm{mM})$ (Scheme 1,4$)$, GSH $(20 \mathrm{mM})$, or L-lysine $(40 \mathrm{mM})$ was then added as a trapping agent, respectively. The total volume of the incubation was $500 \mu \mathrm{l}$. The reactions were initiated by adding NADPH $(1 \mathrm{mM})$. Control samples did not contain NADPH. Reactions were terminated by mixing with ice-cold acetonitrile in a volume of $1: 1$. The resulting mixture was centrifuged at $19,000 \mathrm{~g}$ for 10 minutes at $4^{\circ} \mathrm{C}$ to pellet protein. After the supernatants were evaporated to dryness under nitrogen stream, the reconstituted solution dissolved in $200 \mu l$ acetonitrile:water $(1: 9, \mathrm{v} / \mathrm{v})$ was injected into liquid chromatography-tandem mass spectrometry (LC-MS/MS) for analysis.

The ethyl and vinyl analogs were incubated in the same microsomal system, as described above, to detect possible BBA and GSH adducts, respectively.<smiles>[R]Nc1cccc(CC(=O)NCc2ccc(Br)cc2)c1</smiles>

Scheme 2. Proposed mechanism of ELT metabolic activation. 
Recombinant Human P450 Incubations. ELT was individually incubated with human recombinant P450 enzymes (100 nM), including CYPs 1A2, 2A6, 2B6, 2C9, 2C19, 2D6, 2E1, 3A4, and 3A5. The conditions were the same as the microsomal incubation experiments, except that recombinant P450 enzymes replaced the microsomes. An equal volume of ice-cold acetonitrile that contained propranolol $(500 \mathrm{ng} / \mathrm{ml})$ as internal standard was added to quench the reactions. The experiments were performed in triplicate.

Chemical Synthesis of ELT-BBA and ELT-Lysine Adducts. Synthesis of ELT-BBA adduct was achieved by initial epoxidation of ELT using $m$-CPBA, based on a reported method about peroxidation studies of acetylenes (McDonald and Schwab, 1964). ELT (4 mg, $10.2 \mu \mathrm{mol})$ was dissolved in dichloromethane $(10 \mathrm{ml})$, and $m$-CPBA ( $20 \mathrm{mg}, 116.3 \mu \mathrm{mol})$ and BBA ( $20 \mathrm{mg}, 107.5 \mu \mathrm{mol})$ were then added. After being stirred for 20 hours at room temperature, the reaction mixtures were centrifuged, and the supernatants were condensed under nitrogen stream. The resulting sample was reconstituted with acetonitrile-water (1:9) for LC-MS/MS analysis. Synthesis of ELT-lysine adduct was conducted by the same procedure as described above, except that BBA was replaced by lysine ( $15 \mathrm{mg}, 102.7 \mu \mathrm{mol})$.

P450 3A Inactivation Studies. To determine whether terminal alkyne of ELT is responsible for P450 time-dependent inactivation, experiments were carried out in HLMs with ELT, ethyl, and vinyl analogs as substrates, respectively. Primary incubations $(200 \mu \mathrm{l})$ included one of the substrates $(25$ and $50 \mu \mathrm{M}), 0.5 \mathrm{mg} / \mathrm{ml}$ HLMs, 1 mM NADPH, $3.2 \mathrm{mM} \mathrm{MgCl}_{2}$, and $100 \mathrm{mM}$ PBS (pH 7.4). After incubation at $0,10,20$, and 30 minutes, the primary mixtures $(40 \mu \mathrm{l})$ were transferred to the secondary incubation mixtures $(120 \mu \mathrm{l})$, which included $200 \mu \mathrm{M}$ testosterone, $1 \mathrm{mM}$ $\mathrm{NADPH}, 3.2 \mathrm{mM} \mathrm{MgCl} 2$, and $100 \mathrm{mM}$ PBS ( $\mathrm{pH}$ 7.4). The proportion of dimethylsulfoxide was $0.5 \%(\mathrm{v} / \mathrm{v})$. An equal volume ice-cold acetonitrile containing propranolol as the internal standard was added to terminate the reaction after 15-minute incubation. After centrifugation, the amount of $6 \beta$-hydroxytestosterone produced was measured by LC-MS/MS to determine the remaining CYP3A activity.

Microsomal Protein Modification and Digestion. ELT $(100 \mu \mathrm{M})$ was mixed with $1.0 \mathrm{mg} / \mathrm{ml} \mathrm{HLMs} \mathrm{and} \mathrm{NADPH}(1 \mathrm{mM})$. The final volume of incubation was $0.5 \mathrm{ml}$. After 30 minutes of incubation at $37^{\circ} \mathrm{C}$, the protein samples were denatured by 30 minutes of heating at $60^{\circ} \mathrm{C}$ in a water bath, followed by centrifugation at $19,000 \mathrm{~g}$ for 10 minutes. Ammonium bicarbonate $(50 \mathrm{mM}, \mathrm{pH} 8.0)$ was used to suspend the pellets, which were then mixed with $5.0 \mathrm{mM}$ dithiothreitol. After incubation at $60^{\circ} \mathrm{C}$ for 1 hour, the resulting mixture was digested with a mixture of Pronase $\mathrm{E}$ $(2.5 \mathrm{mg} / \mathrm{ml})$ and chymotrypsin $(2.5 \mathrm{mg} / \mathrm{ml})$ in the presence of $\mathrm{CaCl}_{2}(5.0 \mathrm{mM})$ (Wang et al., 2015). After 15 hours of incubation for $37^{\circ} \mathrm{C}$, the digestion mixtures were centrifuged at $19,000 \mathrm{~g}$ for 10 minutes and the supernatants were analyzed by LC-MS/MS.

To further investigate lysine-based protein adducts, protein digestion was also conducted after the ethyl or vinyl analogs were incubated with HLMs, as described above.

Measurement of Native Heme Loss. The determination of loss of the native heme was achieved, according to the reported literature (Lin et al., 2002). ELT $(50 \mu \mathrm{M})$ or ELT analogs were incubated with $0.5 \mathrm{mg} / \mathrm{ml} \mathrm{HLMs}$ and $1 \mathrm{mM}$ NADPH for 30 minutes at $37^{\circ} \mathrm{C}$. The control samples did not contain NADPH. The final volume of incubation mixtures was $0.5 \mathrm{ml}$. After incubation, the mixtures were extracted with butanone containing $10 \%$ trifluoroacetic acid. The residue was reconstituted in acetonitrile for LC-UV and LC-MS/MS analysis after the extracted solution was evaporated to dryness under nitrogen stream.

Analytical Conditions. Metabolites were characterized on a SCIEX 5500 mass spectrometer (SCIEX, Foster City, CA) equipped with an Agilent 1260 system (Agilent Technologies, Santa Clara, CA). A ZORBAX SB-C18 column $(5.0 \mu \mathrm{m}, 150 \mathrm{~mm} \times$ $4.6 \mathrm{~mm}$; Agilent Technologies) was used to perform HPLC separation. The mobile phase was water and acetonitrile with $0.1 \%$ formic acid. The gradient elutions for analyzing ELT-BBA and ELT-lysine adducts and separating $6 \beta$-hydroxytestosterone were shown in Supplemental Tables 1 and 2, respectively. All of the metabolites were analyzed in a positive mode by multiple reaction monitoring (MRM) scanning via electrospray ionization, based on structurally specific fragmentation: $\mathrm{m} / \mathrm{z} 595 / 479$ and 597/481 for ELT-BBA adduct, $\mathrm{m} / z$ 556/428 for ELT-lysine adduct, $\mathrm{m} / \mathrm{z}$ 305/269 for $6 \beta$-hydroxytestosterone, and $\mathrm{m} / \mathrm{z}, 261 / 116$ for propranolol as internal standard. Precursor ion scanning of $\mathrm{m} / \mathrm{z} 169,171$ was also used for analyzing the ELT-BBA adduct. In addition, SCIEX 4000 Q-Trap (SCIEX) equipped with an Agilent 1260 system was used to analyze the MS/MS fragments of metabolites. MRM coupled with enhanced product ion mode was employed. The optimized mass spectrometric instrument parameters are shown in supporting information (Supplemental Table 3). The Analyst 1.6.2 software was used to analyze all of the data.
For the analysis of heme, chromatographic separation was conducted on a $\mathrm{C} 4$ column $(250 \times 4.6 \mathrm{~mm}, 5 \mu \mathrm{m}$; VYDAC, Hesperia, CA) with solvents including $0.1 \%$ trifluoroacetic acid in water and $0.05 \%$ trifluoroacetic acid in acetonitrile. The gradient was shown in Supplemental Table 4. The eluates were monitored at $400 \mathrm{~nm}$ and Q1MS scanning mode for the heme and possible heme adducts formed in the bioactivation of ELT.

\section{Results}

Enzyme-Dependent ELT-BBA Adduction. ELT was incubated in HLMs supplemented in BBA as a trapping agent. ELT-BBA adduct (Fig. 1 for its structure) eluted at 11.6 minutes was detected by MRM scanning of $\mathrm{m} / \mathrm{z} 595 / 479, \mathrm{~m} / \mathrm{z}, 597 / 481$, and precursor ion scanning of $\mathrm{m} / \mathrm{z} 169, \mathrm{~m} / \mathrm{z}$ 171 (Fig. 1, E-H). The intensity ratios of these two scan modes (MRM and PI) were about $1: 1$, respectively. No peaks at the corresponding retention time were observed in the absence of NADPH (Fig. 1, A-D), demonstrating that the formation of this adduct was NADPH-dependent. The MS/MS spectrum of the ELT-BBA adduct obtained from MRMenhanced product ion scanning of $\mathrm{m} / \mathrm{z} 595 / 479$ or 597/481 showed the characteristic fragment ions containing bromine at $\mathrm{m} / \mathrm{z}$ 169/171 (loss of $\mathrm{C}_{22} \mathrm{H}_{25} \mathrm{~N}_{4} \mathrm{O}_{5}$ ), 479/481 (loss of $\mathrm{C}_{6} \mathrm{H}_{14} \mathrm{O}_{2}$ ), $537 / 539$ (loss of $\mathrm{C}_{3} \mathrm{H}_{7} \mathrm{O}$ ), and fragment ions containing $N$-phenyl quinazolin-4-amine or oxidized carbonyl moiety without bromine at $\mathrm{m} / z, 266$ (loss of $\mathrm{C}_{14} \mathrm{H}_{21} \mathrm{NO}_{3} \mathrm{Br}$ ) and 294 (loss of $\mathrm{C}_{13} \mathrm{H}_{21} \mathrm{NO}_{2} \mathrm{Br}$ ) (Fig. 1I). No such adduct was detected after incubation of the ethyl or vinyl analogs mixed with HLMs. This indicated that the adduct was formed by oxidation of terminal alkyne (Fig. 6, B and C). To further characterize the adduct, ELT was reacted with $m$-CPBA and BBA. After the reaction, a product was detected that showed the same retention time and similar mass spectrum as that of the product formed in HLM incubations with ELT (Fig. 2).

In a separate experiment, the ethyl and vinyl analogs were individually incubated in the HLM incubation system supplemented with GSH as a trapping agent. For the vinyl analog, two GSH conjugates were detected, including one derived from the epoxide (the representative MS/MS scanning of $m / z, 719 / 590,446$ ) (Supplemental Fig. 5A) and the other arising from the quinoneimine metabolite (MS/MS scanning of $m / z 717 / 588,444$ ) (Supplemental Fig. 5B). As expected, only the quinoneimine-derived GSH conjugate (MS/MS scanning of $\mathrm{m} / \mathrm{z}$, 719/590,446) (Supplemental Fig. 6) was detected in microsomal incubations with the ethyl analog.

P450 Enzymes Responsible for Bioactivation of ELT. Recombinant human P450 incubations were carried out for determining which P450 enzymes contribute to the oxidation to form the ELT-BBA adduct. CYPs 3A4 and 3A5 were found to be the major enzymes in contribution to the bioactivation of ELT. CYP3A4 displayed the highest catalytic activity, and the adduct formation by CYP3A5 was approximately $80 \%$ of that induced by CYP3A4. CYPs 2A6 and 2D6 also slightly catalyzed the ELT-BBA formation. The levels of adduct formation by CYPs 2A6 and 2D6 were about $10 \%$ and $13 \%$ of that formed by CYP3A4, respectively (Fig. 3).

Enzyme Inactivation Effects of ELT and ELT Analogs. Microsomal incubation of ELT at concentrations of 25 and $50 \mu \mathrm{M}$ caused dramatic drop in CYP3A activity after 30 minutes by $36 \%(P<0.01)$ and $63 \%(P<0.001)$, respectively. Unlike ELT, the ethyl analog induced a slight activity decrease by only $3 \%$ and $15 \%(P<0.05)$, respectively. The vinyl analog showed similar degree in enzyme inactivation [approximately $9 \%$ and $20 \%(P<0.05)$; Fig. 4]. The observed significant loss of enzyme inactivation effect of ELT after saturation of the alkynyl group to vinyl and ethyl groups suggests that the terminal alkyne of ELT was involved in the P450 inactivation. 

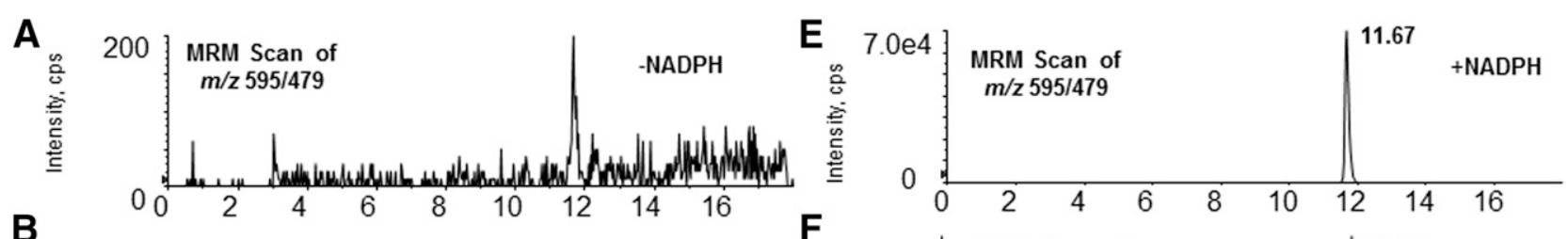

B

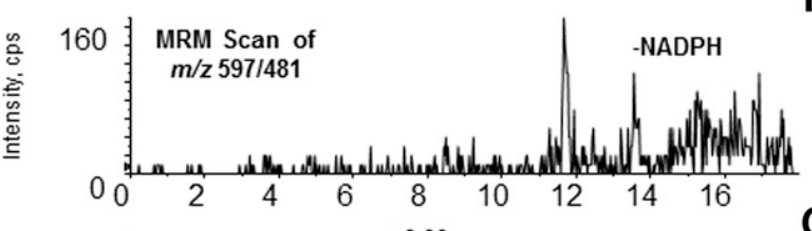

\section{F}
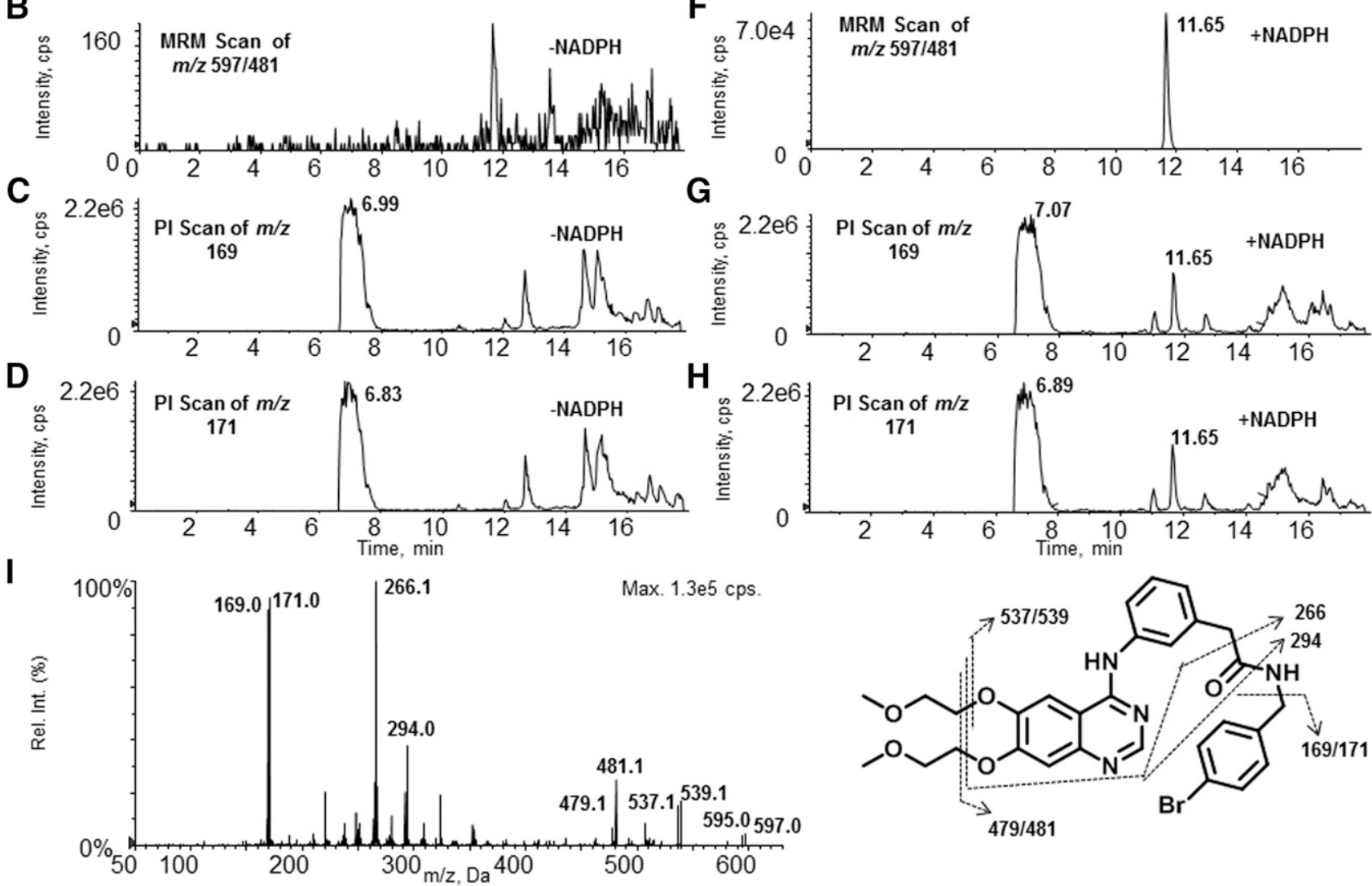

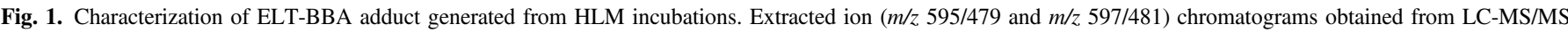

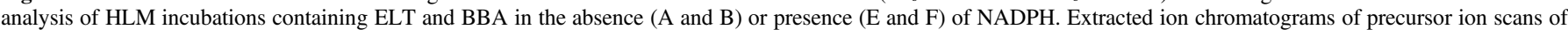

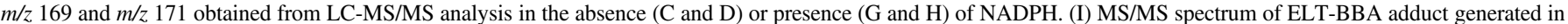
microsomal incubations obtained from LC-MS/MS.

Lysine-Based Protein Modification by ELT. ELT was incubated with HLMs. After incubation, the resulting microsomal protein was exhaustively digested by proteinase and then analyzed by monitoring MRM ion pair $\mathrm{m} / \mathrm{z}, 556 / 428$. A lysine-derived adduct was eluted at
7.7 minutes (Fig. 5D). No such adduct was found in the protein digestion of HLMs in the absence of NADPH (Fig. 5C). The MS/MS characteristic fragment ions of amide moiety of the adduct, such as $\mathrm{m} / \mathrm{z} 410$ and 428 , were detected. Product ion $\mathrm{m} / \mathrm{z} 511$ resulted from the loss of carboxyl group of
A
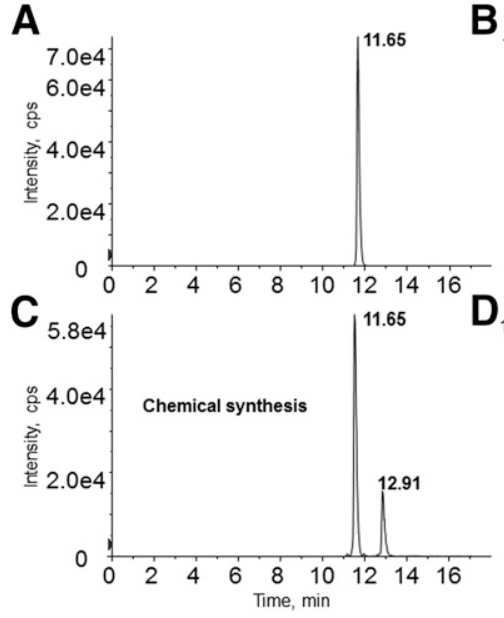
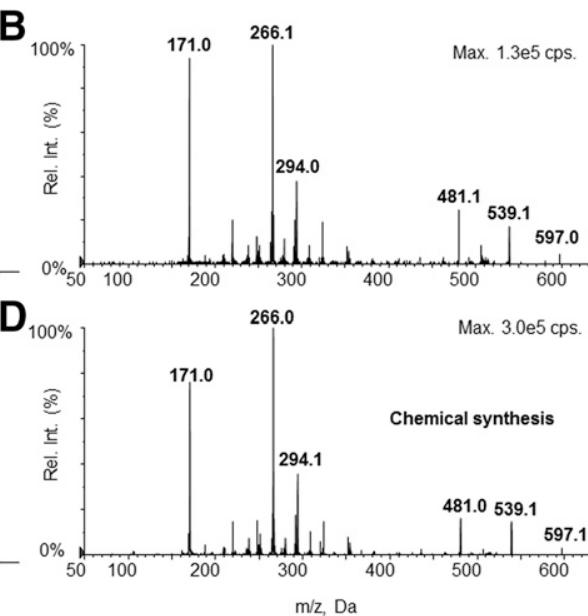

Fig, 2. Characterization of ELT-BBA adduct generated from chemical synthesis. Extracted ion chromatogram of MRM scans of $\mathrm{m} / \mathrm{z}$ 597/481 obtained from microsomal incubations (A) and chemical synthesis (C). MS/MS spectrum of ELT-BBA adduct generated in microsomal incubations (B) and chemical synthesis (D) obtained from LC-MS/MS. 


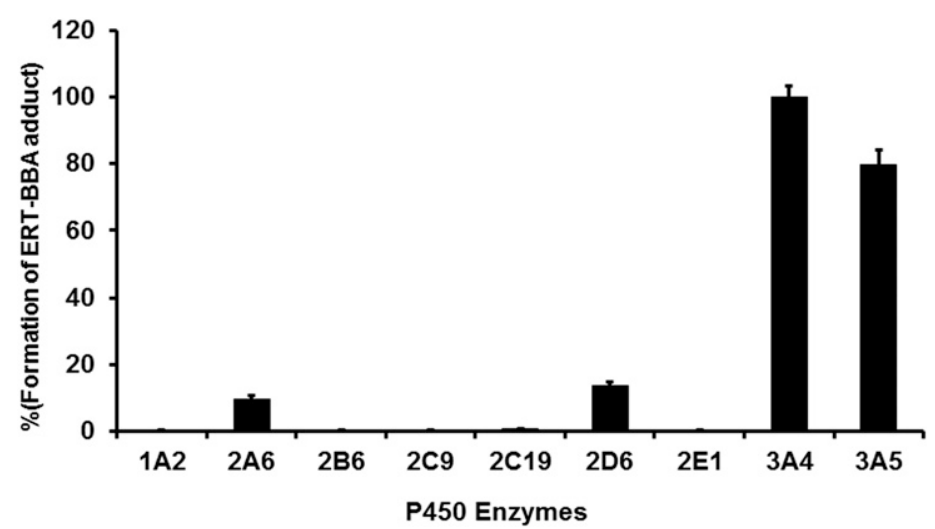

Fig. 3. Formation of ELT-BBA adduct in individual recombinant P450 enzyme incubations containing NADPH, ELT, and BBA. Data represent the mean \pm S.D. $(n=3)$. this adduct (Fig. 5G). Fragment ions $m / z, 266$ and 294 represented the same moieties corresponding to those of the ELT-BBA adduct (Figs. 1I and 5G). The ethyl or vinyl analog was also incubated with HLMs, as described above. No lysine adduct was detected in protein digestion samples (Fig. 6, $\mathrm{E}$ and $\mathrm{F}$ ). An adduct with the same retention time at 7.7 minutes was observed in the HLM incubation in the presence of NADPH and lysine (trapping agent) (Fig. 5B). Synthetic ELT-lysine adduct showed the same chromatographic and mass spectrometry characteristics (Fig. 5, E and F) as that of the corresponding adduct in incubation of HLMs as well as microsomal protein digestion.

Native Heme Loss during Enzyme Inactivation. The native heme of standard solution eluted at approximately 11.8 minutes and was monitored by UV at $400 \mathrm{~nm}$ (Fig. 7A) and showed the $\mathrm{MH}^{+}$ion at $\mathrm{m} / \mathrm{z}$. 616 (Fig. 7B). There was a $\sim 70 \%$ decrease $(P<0.001)$ in native heme of

A
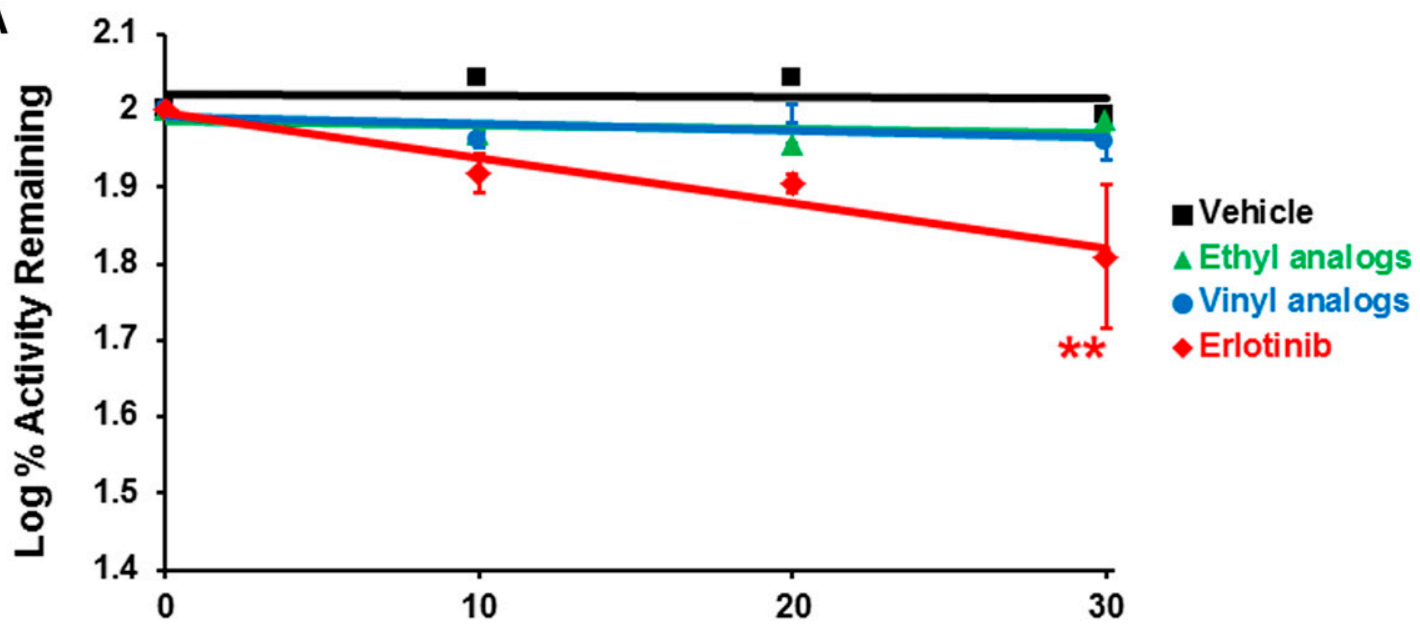

B

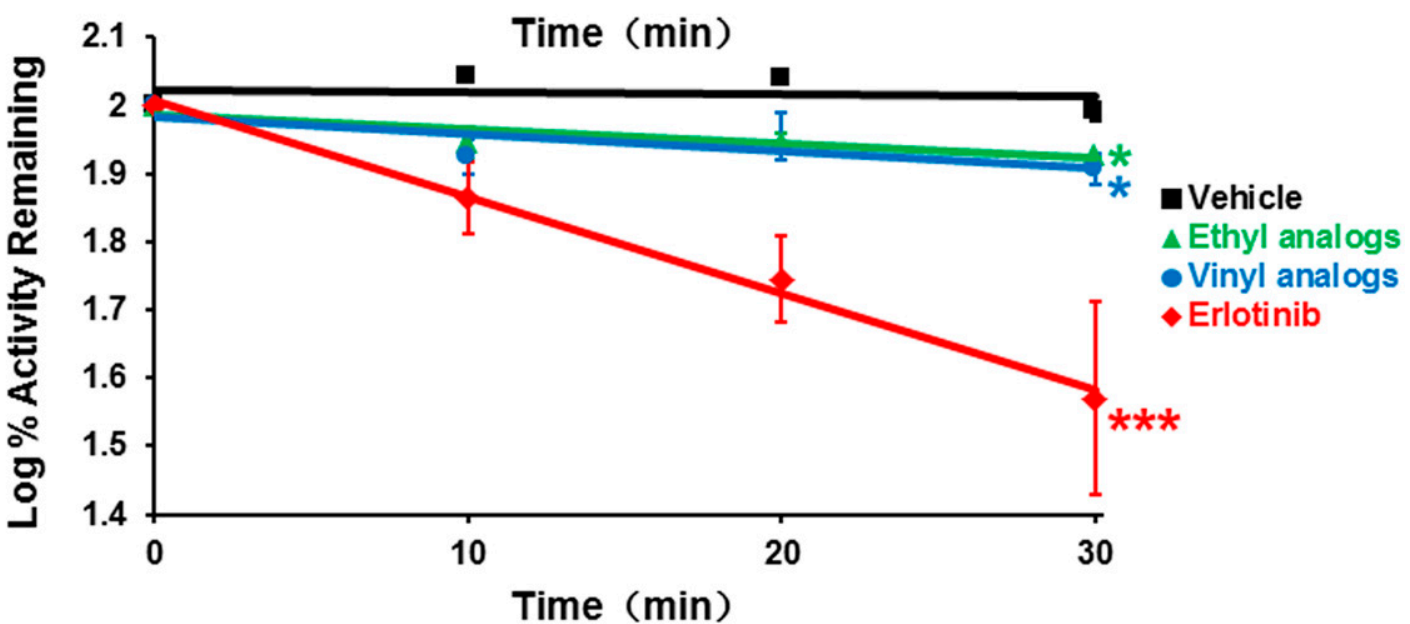

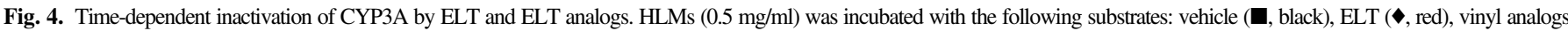

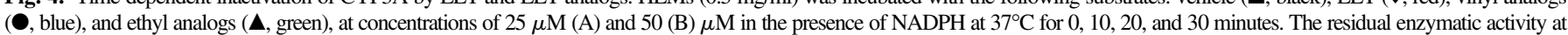

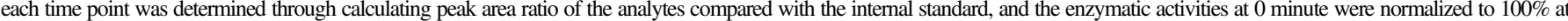
each concentration. Data represent the mean \pm S.D. $(n=3)$. $* P<0.05$; $* * P<0.01 ; * * * P<0.001$ represent Student's $t$ test $P$ values compared with vehicle groups. 

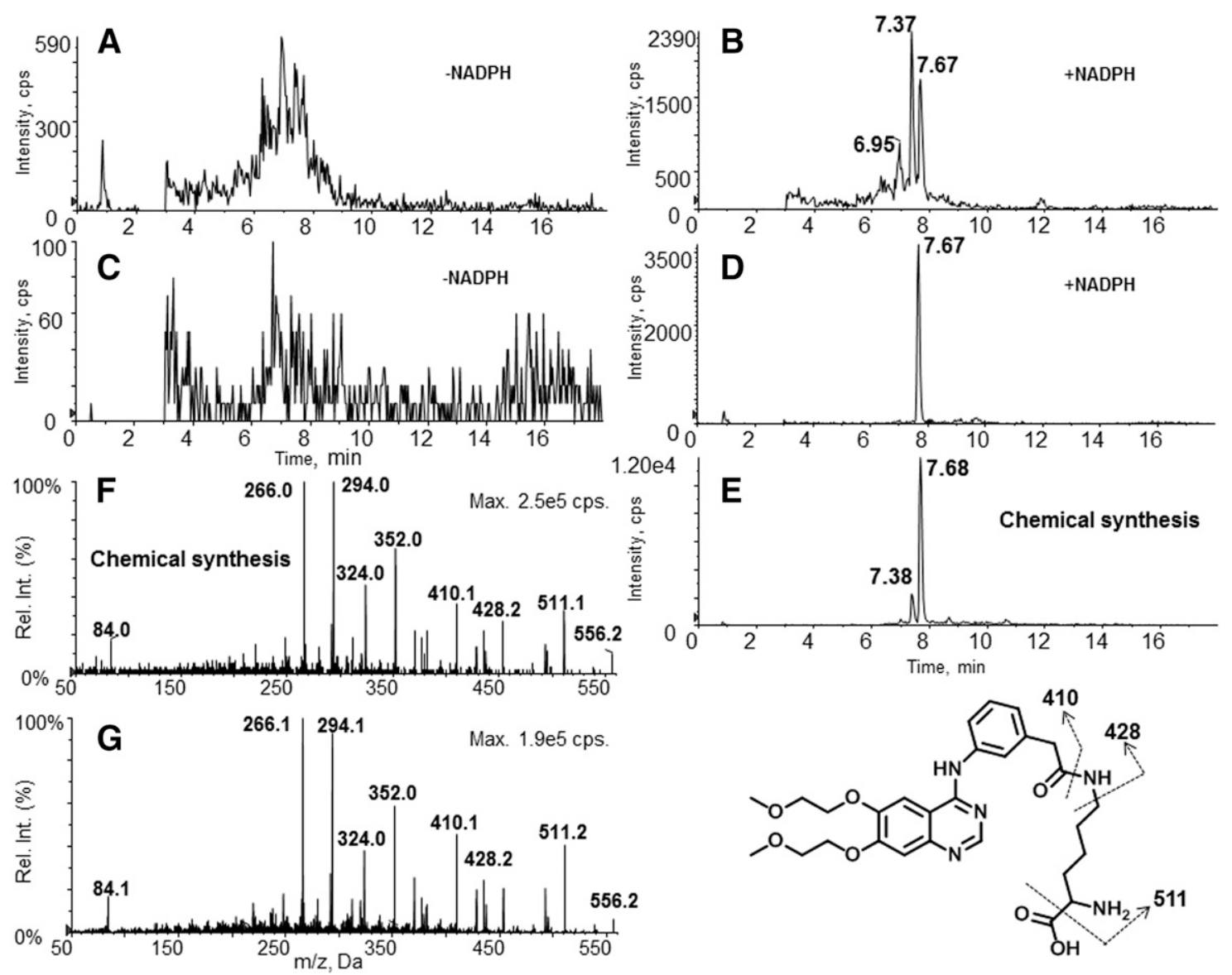

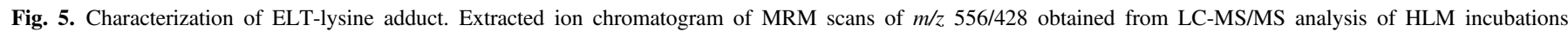

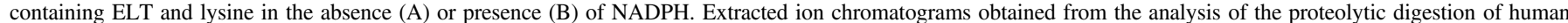

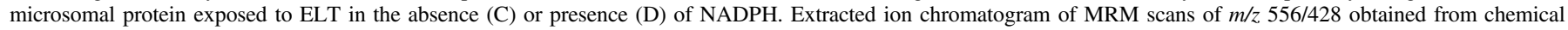
synthesis (E). MS/MS spectrum of ELT-lysine adduct generated in chemical synthesis (F) and proteolytic digestion (G) obtained from LC-MS/MS.

the ELT-inactivated sample (Figs. 7, C and D, and 8). No marked decrease was observed in native heme of both ethyl ( $\sim 5 \%$ decrease)- and vinyl analog ( $\sim 25 \%$ decrease)-incubated samples (Figs. 7, E-H, and 8).

\section{Discussion}

ELT, an EGFR inhibitor used in the treatment of advanced NSCLC, was reported to cause severe liver injury during its clinical use (Liu et al., 2007; Saif, 2008; Huang et al., 2009). ELT can also inhibit CYP3A in a time-dependent manner. Formation of reactive metabolites has been implicated in the mechanism-based inactivation of enzymes and idiosyncratic toxicity. ELT was reportedly biotransformed to a quioneimine metabolite, whereas ketene intermediates were not detected using $\mathrm{N}$-acetyl-lysine as a trapping agent (Li et al., 2010). Despite this, we speculated that the terminal alkyne group of ELT would be oxidized to the corresponding ketene intermediate, which may play a role in CYP3A inactivation. Ketenes are chemically reactive, and unstable, trapping techniques are in need for the metabolite identification. Ketene intermediates reportedly modified $N$-terminal $\alpha$-amino groups of amino acid residues and $\varepsilon$-amino group of lysine residues of apoproteins (Banzon et al., 1995; Chan et al., 2012). Thus, we tested several primary amines as trapping agents, and BBA was found to be the one that is the most effective. Additionally, BBA contained an element bromine, and we took the advantage of its 1:1 natural isotope pattern. This made it easier to monitor targeting analytes by mass spectrometry (Wang et al., 2014), by which we succeeded in detecting ELT-BBA adduct in MRM mode by monitoring of ion pairs $m / z$
595/479, 597/481, and $\mathrm{m} / \mathrm{z}$ 169, 171 (Fig. 1). The observed adduct clearly indicates the production of ELT-derived ketene intermediate. Moreover, one of the three primary metabolism routes of ELT was oxidation of terminal acetylene, followed by hydrolysis to phenyl acetic acid, which was proved to be the stable metabolite of the ketene intermediate (Ling et al., 2006). Formation of the ELT-BBA adduct was found to require the NADPH in the HLM incubation, indicating that P450 enzymes were involved in bioactivation of ELT.

To better understand the metabolic activation of ELT, the ELT-BBA adduct was chemically synthesized by reacting ELT with $m$-CPBA and BBA. $m$-CPBA is an oxidant agent widely used in epoxidation reactions. By analogy to the epoxidation of alkenes, the first product of an acetylene would be an oxirene, and then the reactive ketene was generated by rearrangement of the oxirene (McDonald and Schwab, 1964). Unfortunately, we failed to obtain enough amount of the ELTBBA adduct for NMR characterization, due to the poor yield of the chemical synthesis.

MBI is often referred to as "suicide inactivation." The cytochrome P450s might often be targets of the reactive intermediates generated in the active site of enzyme (Hollenberg et al., 2008). In previous study, ELT has proved to be a mechanism-based inactivator of CYPs 3A4 and 3A5. CYPs $3 \mathrm{~A} 4$ and $1 \mathrm{~A} 1$ were reported to catalyze the formation of the quioneimine metabolite of ELT, but ELT failed to show inhibitory effect on CYP1A1 (Li et al., 2010). The present study demonstrated that both CYPs 3A4 and 3A5 catalyzed the formation of the ketene intermediate 

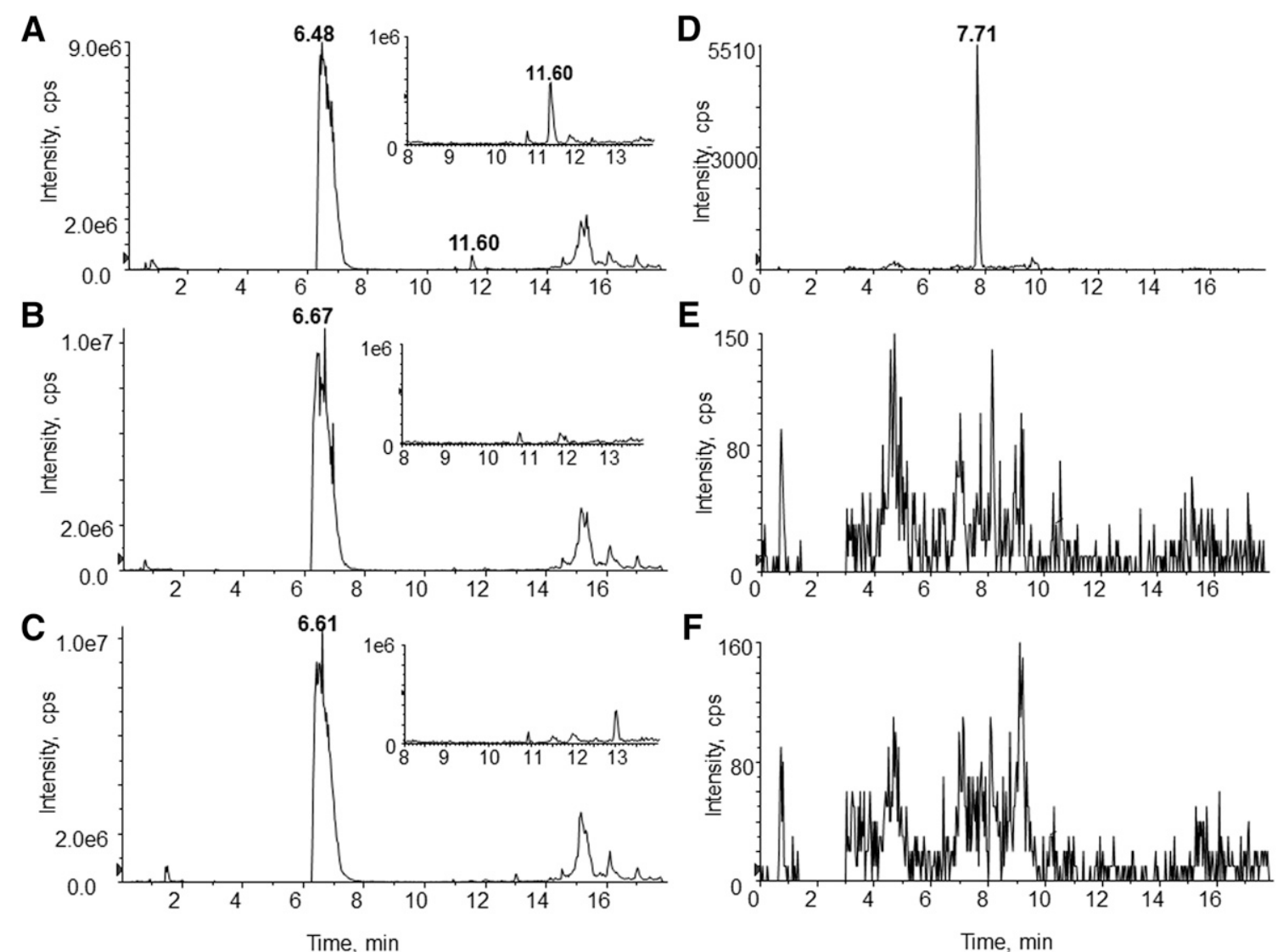

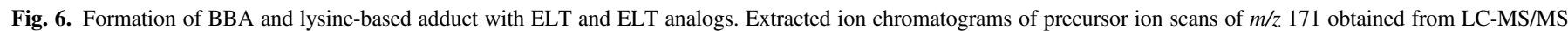

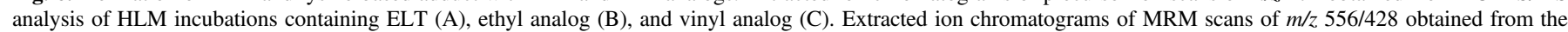
analysis of the proteolytic digestion of human microsomal protein exposed to ELT (D), ethyl analog (E), and vinyl analog (F).

of ELT. It is important to define the role of the two reactive metabolites in the enzyme inactivation. To this end, we replaced the alkyne of ELT with ethyl and vinyl groups and evaluated their inhibitory effects on the enzymes. Both compounds only revealed very weak enzyme inactivation. Structurally, the vinyl group might also be oxidized to epoxide possibly participating in protein modification, due to its constrained
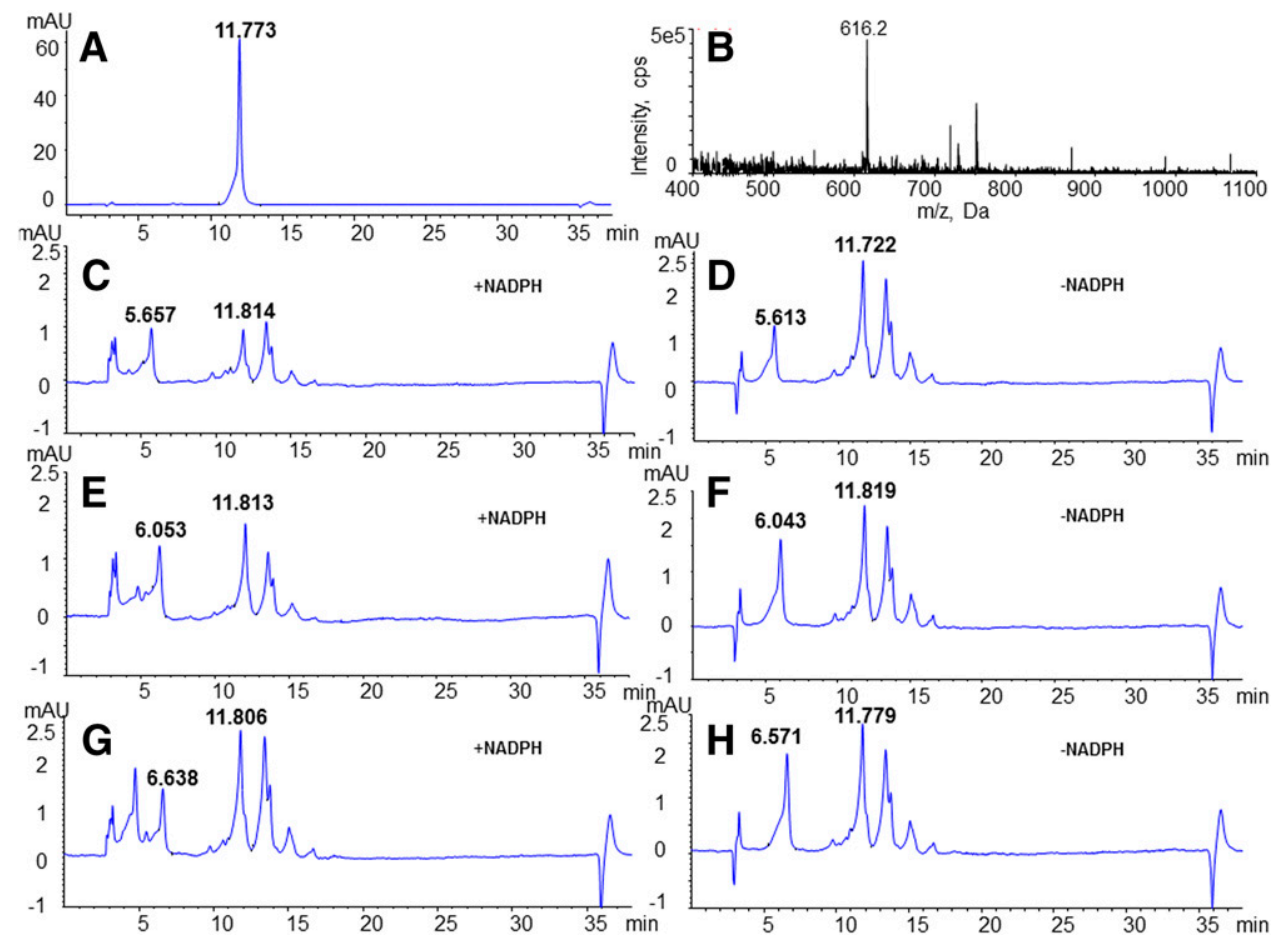

Fig. 7. HPLC profile of the native heme analyses. HPLC chromatogram (A) at $400 \mathrm{~nm}$ and Q1MS spectrum (B) of the standard heme. HPLC chromatograms of HLM incubations containing ELT (C), vinyl analog (E), and ethyl $\operatorname{analog}(\mathrm{G})$ in the presence of NADPH. HPLC chromatograms of HLM incubations containing ELT (D), vinyl analog (F), and ethyl analog (H) in the absence of NADPH. 


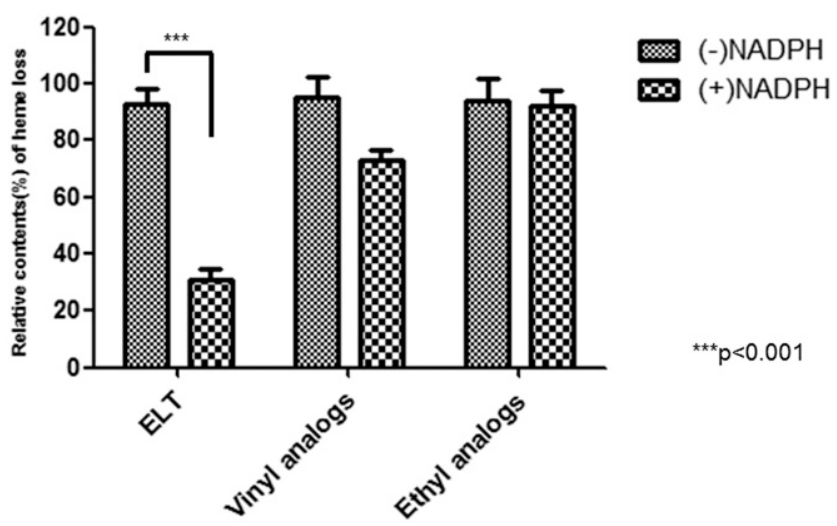

Fig. 8. Native heme loss of ELT, vinyl analogs, and ethyl analogs in the absence and presence of NADPH. Relative contents (\%) of heme loss were determined through calculating peak area of the analytes compared with the vehicle groups. The values of vehicle groups were normalized to $100 \%$. Data represent the mean \pm S.D. $(n=3)$. *** $P<0.001$ represents Student's $t$ test $P$ value.

bond angle (Pearce et al., 2002). To address this possibility, the vinyl analog was incubated in HLMs supplemented with GSH as a trapping agent. Two GSH conjugates were detected by LC-MS/MS after incubation (Supplemental Fig. 5, A and B). One resulted from the corresponding epoxide metabolite, and the other arose from the quinoneimine intermediate ( $\mathrm{Li}$ et al., 2010). Additionally, similar microsomal incubations were carried out to determine the metabolic activation of the ethyl analog. Only one GSH conjugate was detected (Supplemental Fig. 6), and the observed molecular ion of the conjugate matched the mol. wt. of the GSH conjugate derived from the corresponding quinoneimine. The vinyl and ethyl analogs showed little enzyme inhibitory effect (less than 20\%), whereas ELT inactivated more than $60 \%$ of the enzyme in a time-dependent manner. This indicates that the formation of ketene, together with quinoneimine intermediate, contributed to CYP3A inactivation, and the ketene intermediate played the major role.

Previous studies on the drug-induced MBI have led to the suggestion that there are different mechanisms of inactivation via covalent binding to apoprotein and/or destroying the native heme group (Ortiz de Montellano and Komives, 1985; Blobaum, 2006; Lin et al., 2010). Therefore, we analyzed the products of microsomal protein digestion samples pretreated with ELT and measured the relative amount of the native heme loss to better understand the association between bioactivation of ELT and enzyme inactivation. The observed decrease in the amount of the heme after microsomal incubations suggests that the reactive intermediate of ELT would modify the heme, at least partially responsible for the loss of the enzyme activity. Apart from the measurement of the native heme loss, we also attempted to check for the possible heme adducts, using HPLC-UV and mass spectrometry method. No stable heme adduct was detected. We propose that unknown heme fragment or unstable heme adducts were formed in the inactivation reaction so that they cannot be detected. This kind of heme destruction has also been reported by other mechanism-based inactivation studies (Amunugama et al., 2012; Lin et al., 2013). Taken together, our findings suggest that ELT causes inactivation through covalent binding of the bioactivated ELT to the apoprotein of CYPs and the native heme destruction.

Previous clinical studies showed that ELT induced liver injury, which might also be associated with the metabolic activation process. According to the tissue distribution studies (Li et al., 2010), liver was most likely to be toxic target organ, due to accumulated ELT. However, the definitive relationship between the reactive metabolites (ketene and quioneimine) of ELT and liver toxicity remains unknown, although the ketene intermediate played a major role in the enzyme inactivation.
In conclusion, a reactive ketene intermediate was detected in incubations of ELT with HLMs. CYPs 3A4 and 3A5 mainly contributed to the acetylenic bioactivation of ELT. The MBI of the enzymes by ELT was caused by formation of the ketene intermediate. These data are helpful to justify possible drug-drug interactions and to facilitate the understanding of the mechanisms of hepatotoxic action of ELT reported in clinic.

\section{Authorship Contributions}

Participated in research design: Zheng.

Conducted experiments: Zhao, Li, Yang.

Performed data analysis: Zhao, Chen, Peng.

Wrote or contributed to the writing of the manuscript: Zhao, Zheng.

\section{References}

Amunugama HT, Zhang H, and Hollenberg PF (2012) Mechanism-based inactivation of cytochrome P450 2B6 by methadone through destruction of prosthetic heme. Drug Metab Dispos 40: $1765-1770$.

Ban HS, Onagi S, Uno M, Nabeyama W, and Nakamura H (2008) Allene as an alternative functional group for drug design: effect of $\mathrm{C}-\mathrm{C}$ multiple bonds conjugated with quinazolines on the inhibition of EGFR tyrosine kinase. ChemMedChem 3:1094-1103.

Banzon JA, Kuo JM, Miles BW, Fischer DR, Stang PJ, and Raushel FM (1995) Mechanism-based inactivation of phosphotriesterase by reaction of a critical histidine with a ketene intermediate. Biochemistry 34:743-749.

Blobaum AL (2006) Mechanism-based inactivation and reversibility: is there a new trend in the inactivation of cytochrome p450 enzymes? Drug Metab Dispos 34:1-7.

Blobaum AL, Kent UM, Alworth WL, and Hollenberg PF (2002) Mechanism-based inactivation of cytochromes P450 2E1 and 2E1 T303A by tert-butyl acetylenes: characterization of reactive intermediate adducts to the heme and apoprotein. Chem Res Toxicol 15:1561-1571.

Chan AO, Ho CM, Chong HC, Leung YC, Huang JS, Wong MK, and Che CM (2012) Modification of N-terminal $\alpha$-amino groups of peptides and proteins using ketenes. J Am Chem Soc 134:2589-2598.

Dai Q, Ling YH, Lia M, Zou YY, Kroog G, Iwata KK, and Perez-Soler R (2005) Enhanced sensitivity to the HER1/epidermal growth factor receptor tyrosine kinase inhibitor erlotinib hydrochloride in chemotherapy-resistant tumor cell lines. Clin Cancer Res 11:1572-1578.

Hollenberg PF, Kent UM, and Bumpus NN (2008) Mechanism-based inactivation of human cytochromes p450s: experimental characterization, reactive intermediates, and clinical implications. Chem Res Toxicol 21:189-205.

Huang YS, An SJ, Chen ZH, and Wu YL (2009) Three cases of severe hepatic impairment caused by erlotinib. Br J Clin Pharmacol 68:464-467.

Jotte RM and Spigel DR (2015) Advances in molecular-based personalized non-small-cell lung cancer therapy: targeting epidermal growth factor receptor and mechanisms of resistance. Cancer Med 4:1621-1632.

Kalgutkar AS, Obach RS, and Maurer TS (2007) Mechanism-based inactivation of cytochrome P450 enzymes: chemical mechanisms, structure-activity relationships and relationship to clinical drug-drug interactions and idiosyncratic adverse drug reactions. Curr Drug Metab 8:407-447.

Li X, Kamenecka TM, and Cameron MD (2010) Cytochrome P450-mediated bioactivation of the epidermal growth factor receptor inhibitor erlotinib to a reactive electrophile. Drug Metab Dispos 38: $1238-1245$.

Lin HL, D'Agostino J, Kenaan C, Calinski D, and Hollenberg PF (2013) The effect of ritonavir on human CYP2B6 catalytic activity: heme modification contributes to the mechanism-based inactivation of CYP2B6 and CYP3A4 by ritonavir. Drug Metab Dispos 41:1813-1824.

Lin HL and Hollenberg PF (2007) The inactivation of cytochrome P450 3A5 by 17alphaethynylestradiol is cytochrome b5-dependent: metabolic activation of the ethynyl moiety leads to the formation of glutathione conjugates, a heme adduct, and covalent binding to the apoprotein. $J$ Pharmacol Exp Ther 321:276-287.

Lin HL, Kent UM, and Hollenberg PF (2002) Mechanism-based inactivation of cytochrome P450 3A4 by 17 alpha-ethynylestradiol: evidence for heme destruction and covalent binding to protein. J Pharmacol Exp Ther 301:160-167.

Lin HL, Zhang H, Jushchyshyn M, and Hollenberg PF (2010) Covalent modification of Thr302 in cytochrome $\mathrm{P} 4502 \mathrm{~B} 1$ by the mechanism-based inactivator 4-tert-butylphenylacetylene. $J$ Pharmacol Exp Ther 333:663-669.

Ling J, Johnson KA, Miao Z, Rakhit A, Pantze MP, Hamilton M, Lum BL, and Prakash C (2006) Metabolism and excretion of erlotinib, a small molecule inhibitor of epidermal growth factor receptor tyrosine kinase, in healthy male volunteers. Drug Metab Dispos 34:420-426.

Liu W, Makrauer FL, Qamar AA, Jänne PA, and Odze RD (2007) Fulminant hepatic failure secondary to erlotinib. Clin Gastroenterol Hepatol 5:917-920.

Makris D, Scherpereel A, Copin MC, Colin G, Brun L, Lafitte JJ, and Marquette CH (2007) Fatal interstitial lung disease associated with oral erlotinib therapy for lung cancer. BMC Cancer 7:150

Massarelli E, Johnson FM, Erickson HS, Wistuba II, and Papadimitrakopoulou V (2013) Uncommon epidermal growth factor receptor mutations in non-small cell lung cancer and their mechanisms of EGFR tyrosine kinase inhibitors sensitivity and resistance. Lung Cancer 80: 235-241.

McDonald RN and Schwab PA (1964) Strained ring systems. I. Peroxidation studies with certain acetylenes. The relevance of oxirene intermediates. J Am Chem Soc 86:4866-4871.

Orr ST, Ripp SL, Ballard TE, Henderson JL, Scott DO, Obach RS, Sun H, and Kalgutkar AS (2012) Mechanism-based inactivation (MBI) of cytochrome P450 enzymes: structure-activity relationships and discovery strategies to mitigate drug-drug interaction risks. J Med Chem $\mathbf{5 5}$ : 4896-4933.

Ortiz de Montellano PR and Komives EA (1985) Branchpoint for heme alkylation and metabolite formation in the oxidation of arylacetylenes by cytochrome P-450. J Biol Chem 260:3330-3336. Pearce RE, Vakkalagadda GR, and Leeder JS (2002) Pathways of carbamazepine bioactivation in vitro. I. Characterization of human cytochromes P450 responsible for the formation of 2- and 3-hydroxylated metabolites. Drug Metab Dispos 30:1170-1179. 
Saif MW (2008) Erlotinib-induced acute hepatitis in a patient with pancreatic cancer. Clin Adv Hematol Oncol 6:191-199.

Scambia G, Benedetti-Panici P, Ferrandina G, Distefano M, Salerno G, Romanini ME, Fagotti A, and Mancuso S (1995) Epidermal growth factor, oestrogen and progesterone receptor expression in primary ovarian cancer: correlation with clinical outcome and response to chemotherapy. $\mathrm{BrJ}$ Cancer 72:361-366.

Stepan AF, Walker DP, Bauman J, Price DA, Baillie TA, Kalgutkar AS, and Aleo MD (2011) Structural alert/reactive metabolite concept as applied in medicinal chemistry to mitigate the risk of idiosyncratic drug toxicity: a perspective based on the critical examination of trends in the top 200 drugs marketed in the United States. Chem Res Toxicol 24:1345-1410.

Tammaro KA, Baldwin PD, and Lundberg AS (2005) Interstitial lung disease following erlotinib (Tarceva) in a patient who previously tolerated gefitinib (Iressa). J Oncol Pharm Pract 11:127-130.

Veale D, Kerr N, Gibson GJ, Kelly PJ, and Harris AL (1993) The relationship of quantitative epidermal growth factor receptor expression in non-small cell lung cancer to long term survival. Br J Cancer 68:162-165.

von Weymarn LB, Blobaum AL, and Hollenberg PF (2004) The mechanism-based inactivation of p450 2B4 by tert-butyl 1-methyl-2-propynyl ether: structural determination of the adducts to the p450 heme. Arch Biochem Biophys 425:95-105.
Wang K, Li W, Chen J, Peng Y, and Zheng J (2015) Detection of cysteine- and lysine-based protein adductions by reactive metabolites of 2,5-dimethylfuran. Anal Chim Acta 896:93-101. Wang K, Zheng L, Peng Y, Song JE, and Zheng J (2014) Selective and sensitive platform for function-based screening of potentially harmful furans. Anal Chem 86:10755-10762.

Address correspondence to: Dr. Xiaohui Chen, School of Pharmacy, Shenyang Pharmaceutical University, 103 Wenhua Road, Shenyang, Liaoning 110016, P. R. China. E-mail: cxh_syphu@hotmail.com or Dr. Jiang Zheng, State Key Laboratory of Functions and Applications of Medicinal Plants, Key Laboratory of Pharmaceutics of Guizhou Province, Guizhou Medical University, Guiyang, Guizhou 550004, P. R. China; Wuya College of Innovation, Shenyang Pharmaceutical University, 103 Wenhua Road, Shenyang, Liaoning 110016, P. R. China. E-mail: zhengneu@ yahoo.com 